\title{
Control Approach to an Ill-Posed Variational Inequality
}

\author{
G. Marinoschi ${ }^{1 *}$ \\ ${ }^{1}$ Institute of Mathematical Statistics and Applied Mathematics of the Romanian Academy, \\ Calea 13 Septembrie 13, and Simion Stoilow Institute of Mathematics, \\ research group of the project PN-II-ID-PCE-2011-3-0045, Bucharest, Romania
}

\begin{abstract}
We are concerned with the proof of a generalized solution to an ill-posed variational inequality. This is determined as a solution to an appropriate minimization problem involving a nonconvex functional, treated by an optimal control technique.
\end{abstract}

Keywords and phrases: nonlinear parabolic equations, ill-posed problems, optimal control, free boundary problems, variational inequalities, absorption-desorption processes

Mathematics Subject Classification: 35K55, 47J06, 49J20, 76SXX1

\section{Introduction}

In this paper we aim to provide a generalized solution to an ill-posed variational inequality by using an optimal control technique. More exactly, we deal with the problem

$$
\begin{gathered}
y_{t}-\Delta y \in \partial I_{K}(y)+f, \text { in } Q=(0, T) \times \Omega, \\
-\frac{\partial y}{\partial \nu}=g, \quad \text { on } \Sigma=(0, T) \times \Gamma, \\
y(0)=y_{0}, \text { in } \Omega,
\end{gathered}
$$

where $\Omega$ is a bounded open subset of $\mathbb{R}^{N}$, with a $C^{1}$ boundary $\Gamma, T$ is finite, $\frac{\partial}{\partial \nu}$ is the normal derivative to $\Gamma, f, g$ and $y_{0}$ are given in $Q, \Sigma$, and $\Omega$, respectively. All over in the paper, $\phi_{t}$ denotes the time derivative of a generic function $\phi$. Here, $I_{K}$ is the indicator function of a closed convex subset $K$ of $\mathbb{R}$,

$$
I_{K}(r)=0 \text { if } r \in K, I_{K}(r)=+\infty \text { if } r \notin K,
$$

and $\partial I_{K}(r)$ is its subdifferential, that is

$$
\partial I_{K}(r)=\{\eta \in \mathbb{R} ; \eta(r-\bar{r}) \geq 0, \forall \bar{r} \in K\} .
$$

In the following we assume that $K=[b, c], b, c \in \mathbb{R}, b \geq 0, c>0$, and so

$$
\partial I_{K}(r)= \begin{cases}(-\infty, 0], & \text { if } r=b, \\ 0, & \text { if } r \in(b, c), \\ {[0,+\infty),} & \text { if } r=c .\end{cases}
$$

${ }^{*}$ Corresponding author. E-mail: gmarino@acad.ro, gabimarinoschi@yahoo.com 
For a later use, we also introduce the maximal monotone graph

$$
H_{b, c}(r)= \begin{cases}b, & \text { if } r<0, \\ {[b, c],} & \text { if } r=0, \\ c, & \text { if } r>0,\end{cases}
$$

which clearly is the inverse of $\partial I_{K}$.

The model (1.1)-(1.3) may describe an absorption-desorption process, as for example oxygen absorbed by biological tissues (see e.g., [4], [7], [2], p. 140). Also, we can refer to a fluid absorbed into the pores of a porous material (e.g., water in the soil), or radiation or heat retained or ceded by materials or tissues. In this model, $y$ is the absorbed or desorbed fluid concentration at the rate $f$, whenever it is in the interval $(b, c)$.

Equations (1.1)-(1.3) describe in fact the evolution of a physical system in time dependent evolving domains, which can occur as subsets of $\Omega$, namely

$$
\begin{aligned}
\Omega_{b, c}(t) & =\{x \in \Omega ; b<y(t, x)<c\}, \\
\Omega_{b}(t) & =\{x \in \Omega ; y(t, x)=b\}, \Omega_{c}(t)=\{x \in \Omega ; y(t, x)=c\}
\end{aligned}
$$

with the boundaries $\Gamma_{b}(t)=\partial \Omega_{b}(t), \Gamma_{c}(t)=\partial \Omega_{c}(t)$.

Roughly speaking (1.1)-(1.3) reduces to

$$
\begin{gathered}
y_{t}-\Delta y=f \text { in } Q_{b, c}(t)=(0, T) \times \Omega_{b, c}(t), \\
y_{t}-\Delta y \leq f, \text { in } Q_{b}(t)=(0, T) \times \Omega_{b}(t), \\
y_{t}-\Delta y \geq f, \text { in } Q_{c}(t)=(0, T) \times \Omega_{c}(t), \\
-\frac{\partial y}{\partial \nu}=0, \text { on } \Sigma_{b, c}(t)=(0, T) \times \Gamma_{b, c}(t), \\
-\frac{\partial y}{\partial \nu}=g, \text { on } \Sigma=(0, T) \times \Gamma, \\
y(0)=y_{0}, \text { in } \Omega .
\end{gathered}
$$

If $y$ were a smooth solution, then (1.4)-(1.9) would follow in the sense of distributions by a direct calculation. But, problem (1.1)-(1.3) with the maximal monotone graph $\partial I_{K}(y)$ on the right-hand side of equation (1.1) is ill-posed, so it might have only a local in time solution. That is why, as a heuristically observation we can say that in the free boundary problem described by (1.4)-(1.9), the time evolving subset $(0, T) \times \Omega_{c}(t)$ represents the domain where the concentration remains at the high constant level $c$, even if it should still increase due to a positive jump of the supply rate $u+f>f$, the subset $(0, T) \times \Omega_{b}(t)$ represents the inferior limit of the desorption domain, where the concentration remains at the low level $b$, even if it should still decrease due to a supply rate $u+f<f$, and the process evolves such that the fluid concentration remains between the levels $b$ and $c$, at the rate $f$. Here we denoted $u(t, x) \in \partial I_{K}(y(t, x))$ a.e. $(t, x) \in Q$.

Since (1.1)-(1.3) is ill-posed we aim to find, by an optimal control technique, a function $y$ which can be viewed as an approximating solution to (1.1)-(1.3). For solving (1.1)-(1.3) in terms of a control problem one should search for a solution which minimizes the distance between $y$ and the graph $H_{b, c}(u)$, roughly speaking to study the minimization problem

$$
\operatorname{Min}\left\{\frac{\lambda_{1}}{2} \int_{Q}\left(y^{u}(t, x)-H_{b, c}(u(t, x))\right)^{2} d x d t+\frac{\lambda_{2}}{2} \int_{Q} u^{2}(t, x) d x d t\right\},
$$

with the controller $u$. However, in this problem we do not have a sufficient regularity for $u$ to prove existence, so that we have to change the control into another one $v$, and deduce $u$ from an additional equation which guarantees the desired regularity. To this end, we define

$$
J(v, w)=\frac{\lambda_{1}}{2} \int_{Q}\left(y^{v, w}(t, x)-w(t, x)\right)^{2} d x d t+\frac{\lambda_{2}}{2} \int_{Q} v^{2}(t, x) d x d t,
$$


and introduce the following minimization problem for the nonconvex functional $J$,

$$
\operatorname{Min}\left\{J(v, w) ; v \in L^{2}(Q), w \in L^{\infty}(Q), w(t, x) \in H_{b, c}\left(u^{v, w}(t, x)\right) \text { a.e. }(t, x) \in Q\right\},
$$

subject to the system

$$
\begin{aligned}
y_{t}^{v, w}-\Delta y^{v, w}=u^{v, w}+f, \text { in } Q, & \\
-\frac{\partial y^{v, w}}{\partial \nu}=g, & \text { on } \Sigma, \\
y^{v, w}(0)=y_{0}, & \text { in } \Omega \\
u_{t}^{v, w}-\Delta u^{v, w}=v, & \text { in } Q, \\
u^{v, w}=0, & \text { on } \Sigma, \\
u^{v, w}(0)=u_{0}, & \text { in } \Omega .
\end{aligned}
$$

We specify that $u_{0}$ is arbitrary, but fixed. In particular it can be taken 0 .

Problem (1.11) will be treated as a control problem with two controllers, $v$ and $w$. Actually, it can be viewed as a control problem for a singular system (see e.g., appropriate examples in [6]). The functions $u^{v, w}$ and $y^{v, w}$ are the states (indicated by the superscripts ${ }^{v, w}$ ) controlled by $v$ and $w$. For simplicity, later we shall drop these superscripts. The function $u$ is determined by (1.13) in order to get the regularity necessary to prove the existence for (1.11). The positive constants $\lambda_{1}$ and $\lambda_{2}$ can be chosen such that to induce more importance to one term or another in (1.10).

A solution to (1.11) realizes the minimum in $J$, and as a matter of fact if $J$ computed at this solution were zero, then the corresponding state $y$ would satisfy (1.1). Since the existence of a null minimizer cannot be proved, it follows that the state $y$ corresponding to a solution to (1.11) only approximates a section of $H_{b, c}(u)$. Therefore, we call the state $y$ provided by a solution to (1.11) a generalized solution to (1.1)-(1.3). The existence of such a generalized solution, in other words the existence of a minimum of $J$, is proved in Section 2, Theorem 2.1. Since this solution cannot be directly characterized, due to the presence of the graph $H_{b, c}$, we shall introduce in Section 3 an approximating minimization problem (indexed upon $\varepsilon$ ) which converges in some sense to (1.11), as $\varepsilon \rightarrow 0$. The existence of a minimizer $\left\{v_{\varepsilon}^{*}, w_{\varepsilon}^{*}\right\}$ and the convergence result will be given in Section 3, Proposition 3.1 and Theorem 3.2. The calculation of the necessary conditions that should be satisfied by $\left\{v_{\varepsilon}^{*}, w_{\varepsilon}^{*}\right\}$ as a minimizer for the approximating problem will be provided in Section 4, by using a further approximation (indexed on $\sigma$ ) and an adapted penalization method (see [2]).

\section{Existence of the control}

In this section we prove the existence of a solution to the minimization problem (1.11).

We denote by $H^{1}(\Omega)$ and $H^{2}(\Omega)$ the standard Sobolev spaces and by $H_{0}^{1}(\Omega)$ the space of the functions belonging to $H^{1}(\Omega)$ with vanishing traces on the boundary.

We also note that if $v \in L^{2}(Q), u_{0} \in L^{2}(\Omega)$ then (1.13) has a unique solution

$$
u \in W^{1,2}\left([0, T] ; H^{-1}(\Omega)\right) \cap L^{2}\left(0, T ; H_{0}^{1}(\Omega)\right) \cap C\left([0, T] ; L^{2}(\Omega)\right),
$$

and if $f \in L^{2}(Q)$ and $g \in L^{2}(\Sigma)$, problem (1.12) has a unique solution

$$
y \in W^{1,2}\left([0, T] ;\left(H^{1}(\Omega)\right)^{\prime}\right) \cap L^{2}\left(0, T ; H^{1}(\Omega)\right) \cap C\left([0, T] ; L^{2}(\Omega)\right),
$$


where $H^{-1}(\Omega)$ and $\left(H^{1}(\Omega)\right)^{\prime}$ are the dual spaces of $H_{0}^{1}(\Omega)$ and $H^{1}(\Omega)$, respectively.

Theorem 2.1. Let $y_{0} \in L^{2}(\Omega), u_{0} \in L^{2}(\Omega), f \in L^{2}(Q), g \in L^{2}(\Sigma)$. Then, problem (1.11) has at least one solution.

Proof. Let us denote $d=\inf _{v, w} J(v, w)$. Consider a minimizing sequence $\left\{v_{n}, w_{n}\right\}$ such that

$$
d \leq \frac{\lambda_{1}}{2} \int_{Q}\left(y_{n}-w_{n}\right)^{2} d x d t+\frac{\lambda_{2}}{2} \int_{Q} v_{n}^{2} d x d t \leq d+\frac{1}{n}, n \geq 1
$$

where $y_{n}$ and $u_{n}$ are the unique solutions to (1.12)-(1.13) corresponding to $v=v_{n}$. We can select subsequences (denoted still by $n$ ) such that, as $n \rightarrow \infty$, we have

$$
\begin{gathered}
v_{n} \rightarrow v \text { weakly in } L^{2}(Q), \\
w_{n} \rightarrow w \text { weak* in } L^{\infty}(Q) .
\end{gathered}
$$

By (1.13) and (1.12) we deduce that

$$
\begin{aligned}
& u_{n} \rightarrow u \text { weakly in } W^{1,2}\left([0, T] ; H^{-1}(\Omega)\right) \cap L^{2}\left(0, T ; H_{0}^{1}(\Omega)\right), \\
& y_{n} \rightarrow y \text { weakly in } W^{1,2}\left([0, T] ;\left(H^{1}(\Omega)\right)^{\prime}\right) \cap L^{2}\left(0, T ; H^{1}(\Omega)\right),
\end{aligned}
$$

and

$$
u_{n} \rightarrow u, y_{n} \rightarrow y \text { strongly in } L^{2}(Q),
$$

by the Lions theorem (see [5], p. 58). Then, it is obvious that $y$ and $u$ are the solutions to (1.12)-(1.13) corresponding to $v$. We recall that $w_{n}(t, x) \in H_{b, c}\left(u_{n}(t, x)\right)$ a.e. $(t, x) \in Q$, and since the realization of $H_{b, c}$ on $L^{2}(Q)$ is maximal monotone, hence strongly-weakly closed, we get that $w(t, x) \in H_{b, c}(u(t, x))$ a.e. $(t, x) \in Q$. Finally, we pass to the limit in (2.1) and on the basis of the weak lower semicontinuity of the convex integrands we get that $d=J(v, w)$.

\section{The approximating problems}

Since $J$ is not differentiable we cannot determine the optimality conditions directly for (1.11) and we have to introduce an approximating minimization problem in which the graph $H_{b, c}$ is approximated. Next we show its convergence to problem (1.11).

First, we define $j: \mathbb{R} \rightarrow \mathbb{R}$,

$$
j(r)=\left\{\begin{array}{l}
b r, r<0 \\
c r, r \geq 0
\end{array}\right.
$$

and introduce its conjugate $j^{*}: \mathbb{R} \rightarrow \mathbb{R}$, by $j^{*}(\omega)=\sup _{r \in \mathbb{R}}(\omega r-j(r))$, getting that

$$
j^{*}(\omega)=I_{K}(\omega), \text { for any } \omega \in \mathbb{R} .
$$

We note that $\partial j(r)=H_{b, c}(r)$, for all $r \in \mathbb{R}$. We recall that two conjugate functions $j$ and $j^{*}$ satisfy the Legendre-Fenchel relations (see e.g., [3], p. 6)

$$
\begin{aligned}
& j(r)+j^{*}(\omega) \geq r \omega, \text { for all } r, \omega \in R, \\
& j(r)+j^{*}(\omega)=r \omega, \text { iff } \omega \in \partial j(r),
\end{aligned}
$$

where $\partial j$ is the subdifferential of $j$. 
Let $\varepsilon>0$. We define

$$
\begin{aligned}
J_{\varepsilon}(v, w)= & \frac{\lambda_{1}}{2} \int_{Q}(y(t, x)-w(t, x))^{2} d x d t+\frac{\lambda_{2}}{2} \int_{Q} v^{2}(t, x) d x d t \\
& +\frac{1}{\varepsilon} \int_{Q}\left(j\left(u(t, x)+j^{*}(w(t, x))-u(t, x) w(t, x)\right) d x d t,\right.
\end{aligned}
$$

and introduce the approximating minimization problem

$$
\operatorname{Min}\left\{J_{\varepsilon}(v, w) ; v \in L^{2}(Q), w \in L^{\infty}(Q), b \leq w(t, x) \leq c \text { a.e. }(t, x) \in Q\right\}
$$

subject to (1.12)-(1.13).

Roughly speaking, if the minimum in (3.3) were zero, then the last integral in (3.2) would be zero and so, by $(3.1)$ one would obtain that $w(t, x) \in \partial j(u(t, x))=H_{b, c}(u(t, x))$ a.e. $(t, x) \in Q$. The fact that $(3.3)$ is an appropriate approximate for (1.11) is rigorously proved in Theorem 3.2.

It is obvious that, by considering $(3.3)$ we can write $j^{*}(w(t, x))=0$ in $J_{\varepsilon}(v, w)$.

Proposition 3.1. Let $y_{0} \in L^{2}(\Omega), u_{0} \in L^{2}(\Omega), f \in L^{2}(Q), g \in L^{2}(\Sigma)$. Then, problem (3.3) has at least one solution.

Proof. Let us denote $d_{\varepsilon}=\inf _{v, w} J_{\varepsilon}(v, w)$ and consider a minimizing sequence $\left\{v_{\varepsilon}^{n}, w_{\varepsilon}^{n}\right\}$ such that

$$
\begin{aligned}
d \leq & \frac{\lambda_{1}}{2} \int_{Q}\left(y_{\varepsilon}^{n}-w_{\varepsilon}^{n}\right)^{2} d x d t+\frac{\lambda_{2}}{2} \int_{Q}\left(v_{\varepsilon}^{n}\right)^{2} d x d t \\
& +\frac{1}{\varepsilon} \int_{Q}\left(j\left(u_{\varepsilon}^{n}\right)-u_{\varepsilon}^{n} w_{\varepsilon}^{n}\right) d x d t \leq d+\frac{1}{n},
\end{aligned}
$$

where $y_{\varepsilon}^{n}$ and $u_{\varepsilon}^{n}$ are the unique solutions to (1.12)-(1.13) corresponding to $v=v_{\varepsilon}^{n}$. As in Theorem 2.1, Section 2 , it follows on subsequences $(n \rightarrow \infty)$ that

$$
\begin{gathered}
v_{\varepsilon}^{n} \rightarrow v_{\varepsilon} \text { weakly in } L^{2}(Q), \\
w_{\varepsilon}^{n} \rightarrow w_{\varepsilon} \text { weak }^{*} \text { in } L^{\infty}(Q), w_{\varepsilon}(t, x) \in[b, c] \text { a.e. }(t, x) \in Q .
\end{gathered}
$$

By (1.13) and (1.12) we deduce

$$
\begin{gathered}
u_{\varepsilon}^{n} \rightarrow u_{\varepsilon} \text { weakly in } W^{1,2}\left([0, T] ; H^{-1}(\Omega)\right) \cap L^{2}\left(0, T ; H_{0}^{1}(\Omega)\right), \\
y_{\varepsilon}^{n} \rightarrow y_{\varepsilon} \text { weakly in } W^{1,2}\left([0, T] ;\left(H^{1}(\Omega)\right)^{\prime}\right) \cap L^{2}\left(0, T ; H^{1}(\Omega)\right), \\
u_{\varepsilon}^{n} \rightarrow u_{\varepsilon}, y_{\varepsilon}^{n} \rightarrow y_{\varepsilon} \text { strongly in } L^{2}(Q),
\end{gathered}
$$

and $y_{\varepsilon}$ and $u_{\varepsilon}$ are the solutions to (1.12)-(1.13) corresponding to $v_{\varepsilon}$. Then,

$$
\lim _{n \rightarrow \infty} \int_{Q} y_{\varepsilon}^{n} w_{\varepsilon}^{n} d x d t=\int_{Q} y_{\varepsilon} w_{\varepsilon} d x d t
$$

and since $j$ is continuous and $u_{\varepsilon}^{n} \rightarrow u_{\varepsilon}$ a.e. in $Q$, we have $j\left(u_{\varepsilon}^{n}(t, x)\right) \rightarrow j\left(u_{\varepsilon}(t, x)\right)$, a.e., as $n \rightarrow \infty$. Now, we pass to the limit in (3.4). In order to be allowed to apply the Fatou lemma we recall first that a proper, convex l.s.c. function is bounded below by an affine function (see [3], p. 5), hence

$$
j(t, x, r) \geq k_{1}(t, x) r+k_{2}(t, x), k_{1}, k_{2} \in L^{\infty}(Q) .
$$

We replace $j$ in (3.4) by $\widetilde{j}(r)=j(r)-k_{1} r-k_{2}$, which is nonnegative, and have

$$
\begin{aligned}
& d+\frac{1}{\varepsilon} \int_{Q} u_{\varepsilon}^{n} w_{\varepsilon}^{n} d x d t-\int_{Q}\left(k_{1} u_{\varepsilon}^{n}+k_{2}\right) d x d t \\
\leq & \frac{\lambda_{1}}{2} \int_{Q}\left(y_{\varepsilon}^{n}-w_{\varepsilon}^{n}\right)^{2} d x d t+\frac{\lambda_{2}}{2} \int_{Q}\left(v_{\varepsilon}^{n}\right)^{2} d x d t \\
& +\frac{1}{\varepsilon} \int_{Q} \widetilde{j}\left(u_{\varepsilon}^{n}\right) d x d t \leq d+\frac{1}{n}+\frac{1}{\varepsilon} \int_{Q} u_{\varepsilon}^{n} w_{\varepsilon}^{n} d x d t-\int_{Q}\left(k_{1} u_{\varepsilon}^{n}+k_{2}\right) d x d t .
\end{aligned}
$$


We pass to the limit as $n \rightarrow \infty$, applying the Fatou lemma and finally getting that $d_{\varepsilon}=J_{\varepsilon}\left(v_{\varepsilon}, w_{\varepsilon}\right)$. This proves that $\left\{v_{\varepsilon}, w_{\varepsilon}\right\}$ is a minimizer for $J_{\varepsilon}$.

Next we should deal with the optimality conditions for problem (3.3), which are the necessary conditions that the optimal controller $\left\{v_{\varepsilon}^{*}, w_{\varepsilon}^{*}\right\}$ must satisfy in order to be a solution to (3.3). Because $J_{\varepsilon}$ is not differentiable due to $j$, we shall introduce a second approximating problem in order to regularize $j$. This is based on the Moreau regularization defined as

$$
j_{\sigma}(r)=\inf _{s \in \mathbb{R}}\left\{\frac{|r-s|^{2}}{2 \sigma}+j(s)\right\}, \text { for any } r \in \mathbb{R}, \sigma>0,
$$

which can be still written

$$
j_{\sigma}(r)=\frac{1}{2 \sigma}\left|(I+\sigma \partial j)^{-1} r-r\right|^{2}+j\left((I+\sigma \partial j)^{-1} r\right),
$$

where $I$ is the identity on $\mathbb{R}$. The function $j_{\sigma}$ is convex, Lipschitz continuous and has the properties (see [3], p. 48)

$$
\begin{aligned}
j_{\sigma}(r) & \leq j(r), \text { for any } r \\
\lim _{\sigma \rightarrow 0} j_{\sigma}(r) & =j(r), \text { for any } r
\end{aligned}
$$

Let $\left\{v_{\varepsilon}^{*}, w_{\varepsilon}^{*}\right\}$ be optimal in $(3.3)$.

We introduce the approximating adapted penalized problem (see [2])

$$
\begin{aligned}
& \operatorname{Min}\left\{J_{\varepsilon, \sigma}(u, v, \eta)=\frac{\lambda_{1}}{2} \int_{Q}(y(t, x)-w(t, x))^{2} d x d t+\frac{\lambda_{2}}{2} \int_{Q} v^{2}(t, x) d x d t\right. \\
& +\frac{1}{\varepsilon} \int_{Q}\left(j_{\sigma}(u(t, x))-u(t, x) w(t, x)\right) d x d t \\
& \left.+\frac{1}{2} \int_{Q}\left(v(t, x)-v_{\varepsilon}^{*}(t, x)\right)^{2} d x d t+\frac{1}{2} \int_{Q}\left(w(t, x)-w_{\varepsilon}^{*}(t, x)\right)^{2} d x d t\right\}
\end{aligned}
$$

subject to (1.12)-(1.13), for all $v \in L^{2}(Q), w \in L^{\infty}(Q), w(t, x) \in[b, c]$ a.e. $(t, x) \in Q$.

It is immediate (by a similar proof as in Proposition 3.1) that problem (3.8) has at least one solution. Next, we prove that this problem indexed upon $\sigma$ converges to the approximating problem indexed upon $\varepsilon$, as $\sigma \rightarrow 0$.

Theorem 3.2. Let $\left(v_{\varepsilon}^{*}, w_{\varepsilon}^{*}\right)$ be optimal in $(3.3)$ and $\left(v_{\varepsilon, \sigma}^{*}, w_{\varepsilon, \sigma}^{*}\right)$ be optimal in (3.8). Then,

$$
\begin{gathered}
v_{\varepsilon, \sigma}^{*} \rightarrow v_{\varepsilon}^{*} \text { strongly in } L^{2}(Q) \text {, as } \sigma \rightarrow 0, \\
w_{\varepsilon, \sigma}^{*} \rightarrow w_{\varepsilon}^{*} \text { strongly in } L^{2}(Q) \text {, as } \sigma \rightarrow 0,
\end{gathered}
$$

and the corresponding states $\left\{u_{\varepsilon, \sigma}^{*}, y_{\varepsilon, \sigma}^{*}\right\}_{\sigma}$ converge to $\left\{u_{\varepsilon}^{*}, y_{\varepsilon}^{*}\right\}$, the corresponding states in (3.3),

$$
\begin{aligned}
u_{\varepsilon, \sigma}^{*} \rightarrow & u_{\varepsilon}^{*} \text { weakly in } W^{1,2}\left([0, T] ; H^{-1}(\Omega)\right) \cap L^{2}\left(0, T ; H_{0}^{1}(\Omega)\right), \\
& \text { strongly in } L^{2}\left(0, T ; L^{2}(\Omega)\right), \\
y_{\varepsilon, \sigma}^{*} \rightarrow y_{\varepsilon}^{*} & \text { weakly in } W^{1,2}\left([0, T] ;\left(H^{1}(\Omega)\right)^{\prime}\right) \cap L^{2}\left(0, T ; H^{1}(\Omega)\right), \\
& \text { strongly in } L^{2}\left(0, T ; L^{2}(\Omega)\right) .
\end{aligned}
$$


Proof. We write that $\left(v_{\varepsilon, \sigma}^{*}, w_{\varepsilon, \sigma}^{*}\right)$ is optimal in (3.8), that is

$$
\begin{aligned}
& \frac{\lambda_{1}}{2} \int_{Q}\left(y_{\varepsilon, \sigma}^{*}-w_{\varepsilon, \sigma}^{*}\right)^{2} d x d t+\frac{\lambda_{2}}{2} \int_{Q}\left(v_{\varepsilon, \sigma}^{*}\right)^{2} d x d t \\
& +\frac{1}{\varepsilon} \int_{Q}\left(j_{\sigma}\left(u_{\varepsilon, \sigma}^{*}\right)-w_{\varepsilon, \sigma}^{*} u_{\varepsilon, \sigma}^{*}\right) d x d t \\
& +\frac{1}{2} \int_{Q}\left(v_{\varepsilon, \sigma}^{*}-v_{\varepsilon}^{*}\right)^{2} d x d t+\frac{1}{2} \int_{Q}\left(w_{\varepsilon, \sigma}^{*}-w_{\varepsilon}^{*}\right)^{2} d x d t \\
\leq & \frac{\lambda_{1}}{2} \int_{Q}(y-w)^{2} d x d t+\frac{\lambda_{2}}{2} \int_{Q} v^{2} d x d t+\frac{1}{\varepsilon} \int_{Q}\left(j_{\sigma}(u)-w u\right) d x d t \\
& +\frac{1}{2} \int_{Q}\left(v-v_{\varepsilon}^{*}\right)^{2} d x d t+\frac{1}{2} \int_{Q}\left(w-w_{\varepsilon}^{*}\right)^{2} d x d t,
\end{aligned}
$$

for all $v \in L^{2}(Q), w \in L^{\infty}(Q), w(t, x) \in[b, c]$ a.e., with $y, u$ the solutions to (1.12)-(1.13) corresponding to $v$.

In particular, we set $v=v_{\varepsilon}^{*}, w=w_{\varepsilon}^{*}$ which provide by (1.12)-(1.13) the optimal states $y_{\varepsilon}^{*}$ and $u_{\varepsilon}^{*}$. We have

$$
\begin{aligned}
& \frac{\lambda_{1}}{2} \int_{Q}\left(y_{\varepsilon, \sigma}^{*}-w_{\varepsilon, \sigma}^{*}\right)^{2} d x d t+\frac{\lambda_{2}}{2} \int_{Q}\left(v_{\varepsilon, \sigma}^{*}\right)^{2} d x d t \\
& +\frac{1}{\varepsilon} \int_{Q}\left(j_{\sigma}\left(u_{\varepsilon, \sigma}^{*}\right)-w_{\varepsilon, \sigma}^{*} u_{\varepsilon, \sigma}^{*}\right) d x d t \\
& +\frac{1}{2} \int_{Q}\left(v_{\varepsilon, \sigma}^{*}-v_{\varepsilon}^{*}\right)^{2} d x d t+\frac{1}{2} \int_{Q}\left(w_{\varepsilon, \sigma}^{*}-w_{\varepsilon}^{*}\right)^{2} d x d t \\
\leq & \frac{\lambda_{1}}{2} \int_{Q}\left(y_{\varepsilon}^{*}-w_{\varepsilon}^{*}\right)^{2} d x d t+\frac{\lambda_{2}}{2} \int_{Q}\left(v_{\varepsilon}^{*}\right)^{2} d x d t+\frac{1}{\varepsilon} \int_{Q}\left(j_{\sigma}\left(u_{\varepsilon}^{*}\right)-w_{\varepsilon}^{*} u_{\varepsilon}^{*}\right) d x d t \\
\leq & \frac{\lambda_{1}}{2} \int_{Q}\left(y_{\varepsilon}^{*}-w_{\varepsilon}^{*}\right)^{2} d x d t+\frac{\lambda_{2}}{2} \int_{Q}\left(v_{\varepsilon}^{*}\right)^{2} d x d t+\frac{1}{\varepsilon} \int_{Q}\left(j\left(u_{\varepsilon}^{*}\right)-w_{\varepsilon}^{*} u_{\varepsilon}^{*}\right) d x d t
\end{aligned}
$$

by (3.7). The left-hand side is bounded independently of $\sigma$, because $u_{\varepsilon}^{*}$ is bounded in $L^{2}(Q)$, by (1.13). Consequently, by selecting subsequences (denoted still by the subscript $\sigma$ ) we get

$$
\begin{gathered}
v_{\varepsilon, \sigma}^{*} \rightarrow v_{\varepsilon} \text { weakly in } L^{2}(Q), \\
w_{\varepsilon, \sigma}^{*} \rightarrow w_{\varepsilon} \text { weak* in } L^{\infty}(Q), \\
u_{\varepsilon, \sigma}^{*} \rightarrow u_{\varepsilon} \text { weakly in } W^{1,2}\left([0, T] ; H^{-1}(\Omega)\right) \cap L^{2}\left(0, T ; H_{0}^{1}(\Omega)\right), \\
y_{\varepsilon, \sigma}^{*} \rightarrow y_{\varepsilon} \text { weakly in } W^{1,2}\left([0, T] ;\left(H^{1}(\Omega)\right)^{\prime}\right) \cap L^{2}\left(0, T ; H^{1}(\Omega)\right), \\
u_{\varepsilon, \sigma}^{*} \rightarrow u_{\varepsilon}, y_{\varepsilon, \sigma}^{*} \rightarrow y_{\varepsilon} \text { strongly in } L^{2}(Q),
\end{gathered}
$$

where $\left\{y_{\varepsilon}, u_{\varepsilon}\right\}$ is the solution to (1.12)-(1.13) corresponding to $v_{\varepsilon}$.

Now, we prove that

$$
\liminf _{\sigma \rightarrow 0} \int_{Q} j_{\sigma}\left(u_{\varepsilon, \sigma}^{*}\right) d x d t \geq \int_{Q} j\left(u_{\varepsilon}\right) d x d t,
$$

where $u_{\varepsilon}=\lim _{\sigma \rightarrow 0} u_{\varepsilon, \sigma}^{*}$. By (3.6) we have

$$
\frac{1}{2 \sigma} \int_{Q}\left|(I+\sigma \partial j)^{-1} u_{\varepsilon, \sigma}^{*}-u_{\varepsilon, \sigma}^{*}\right|^{2} d x d t \leq \int_{Q} j_{\sigma}\left(u_{\varepsilon, \sigma}^{*}\right) d x d t \leq \text { constant },
$$


by (3.12), which implies that

$$
\lim _{\sigma \rightarrow 0}\left\|(I+\sigma \partial j)^{-1} u_{\varepsilon, \sigma}^{*}-u_{\varepsilon, \sigma}^{*}\right\|_{L^{2}(Q)}=0
$$

We deduce that

$$
\lim _{\sigma \rightarrow 0}(I+\sigma \partial j)^{-1} u_{\varepsilon, \sigma}^{*}=u_{\varepsilon} \text { strongly in } L^{2}(Q) .
$$

Again by (3.6) we can write

$$
\liminf _{\sigma \rightarrow 0} \int_{Q} j_{\sigma}\left(u_{\varepsilon, \sigma}^{*}\right) d x d t \geq \liminf _{\sigma \rightarrow 0} \int_{Q} j\left((I+\sigma \partial j)^{-1} u_{\varepsilon, \sigma}^{*}\right) d x d t=\int_{Q} j\left(u_{\varepsilon}\right) d x d t,
$$

by Fatou's lemma (following the argument given in Proposition 3.1), the continuity of $j$ and (3.14). Here $I$ is the identity on $L^{2}(Q)$.

Then, passing to the limit (in the first and last terms) in (3.12), as $\sigma \rightarrow 0$ we get

$$
\begin{aligned}
& \frac{\lambda_{1}}{2} \int_{Q}\left(y_{\varepsilon}-w_{\varepsilon}\right)^{2} d x d t+\frac{\lambda_{2}}{2} \int_{Q}^{2} v_{\varepsilon}^{2} d x d t+\frac{1}{\varepsilon} \int_{Q}\left(j\left(u_{\varepsilon}\right)-w_{\varepsilon} u_{\varepsilon}\right) d x d t \\
& +\frac{1}{2} \int_{Q}\left(v_{\varepsilon}-v_{\varepsilon}^{*}\right)^{2} d x d t+\frac{1}{2} \int_{Q}\left(w_{\varepsilon}-w_{\varepsilon}^{*}\right)^{2} d x d t \\
\leq & \frac{\lambda_{1}}{2} \int_{Q}\left(y_{\varepsilon}^{*}-w_{\varepsilon}^{*}\right)^{2} d x d t+\frac{\lambda_{2}}{2} \int_{Q}\left(v_{\varepsilon}^{*}\right)^{2} d x d t+\frac{1}{\varepsilon} \int_{Q}\left(j\left(u_{\varepsilon}^{*}\right)-w_{\varepsilon}^{*} u_{\varepsilon}^{*}\right) d x d t \\
\leq & \frac{\lambda_{1}}{2} \int_{Q}\left(y_{\varepsilon}-w_{\varepsilon}\right)^{2} d x d t+\lambda_{2} \int_{Q} v_{\varepsilon}^{2} d x d t+\frac{1}{\varepsilon} \int_{Q}\left(j\left(u_{\varepsilon}\right)-w_{\varepsilon} u_{\varepsilon}\right) d x d t .
\end{aligned}
$$

The last inequality can be written because $\left(v_{\varepsilon}^{*}, w_{\varepsilon}^{*}\right)$ is optimal in $(3.3)$. From here we get that

$$
v_{\varepsilon}=v_{\varepsilon}^{*}, w_{\varepsilon}=w_{\varepsilon}^{*} \text { a.e. on } Q,
$$

and so $u_{\varepsilon}=u_{\varepsilon}^{*}$ and $y_{\varepsilon}=y_{\varepsilon}^{*}$ a.e. on $Q$. Actually, by (3.12) we obtain (3.9) and the strong convergences in (3.10)-(3.11). This ends the proof.

\section{Optimality conditions}

Let $\left\{v_{\varepsilon, \sigma}^{*}, w_{\varepsilon, \sigma}^{*}\right\}$ (with the corresponding $\left\{u_{\varepsilon, \sigma}^{*}, y_{\varepsilon, \sigma}^{*}\right\}$ ) be a solution to (3.8) and $\left\{v_{\varepsilon}^{*}, w_{\varepsilon}^{*}\right\}$ (with the corresponding $\left.\left\{u_{\varepsilon}^{*}, y_{\varepsilon}^{*}\right\}\right)$ be a solution to (3.3).

Proposition 4.1. Let $y_{0} \in L^{2}(\Omega), u_{0} \in H_{0}^{1}(\Omega), f \in L^{2}(Q), g \in L^{2}(\Sigma)$ and let $\left\{v_{\varepsilon, \sigma}^{*}, w_{\varepsilon, \sigma}^{*}\right\}$ be an optimal controller in (3.8). Then, the optimality conditions for (3.8) read as

$$
\begin{gathered}
w_{\varepsilon, \sigma}^{*}(t, x)= \begin{cases}b, & \text { in }\left\{(t, x) ; a_{\varepsilon, \sigma}^{*}(t, x) \leq b\right\}, \\
a_{\varepsilon, \sigma}^{*}(t, x), & \text { in }\left\{(t, x) ; b<a_{\varepsilon, \sigma}^{*}(t, x)<c\right\}, \\
c, & \text { in }\left\{(t, x) ; a_{\varepsilon, \sigma}^{*}(t, x) \geq c\right\},\end{cases} \\
v_{\varepsilon, \sigma}^{*}(t, x)=\frac{1}{1+\lambda_{2}}\left(v_{\varepsilon}^{*}+z_{\varepsilon, \sigma}^{*}\right)(t, x),
\end{gathered}
$$

where

$$
a_{\varepsilon, \sigma}^{*}(t, x)=\frac{1}{1+\lambda_{1}}\left(\lambda_{1} y_{\varepsilon, \sigma}^{*}+\frac{1}{\varepsilon} u_{\varepsilon, \sigma}^{*}+w_{\varepsilon}^{*}\right)(t, x)
$$


$z_{\varepsilon, \sigma}^{*}$ is the solution to

$$
\begin{aligned}
\left(z_{\varepsilon, \sigma}^{*}\right)_{t}+\Delta z_{\varepsilon, \sigma}^{*} & =p_{\varepsilon, \sigma}+\frac{1}{\varepsilon}\left(\xi_{\varepsilon, \sigma}^{*}-w_{\varepsilon, \sigma}^{*}\right), & & \text { in } Q, \\
z_{\varepsilon, \sigma}^{*} & =0, & & \text { on } \Sigma, \\
\left(z_{\varepsilon, \sigma}^{*}\right)(T) & =0, & & \text { in } \Omega, \\
\xi_{\varepsilon, \sigma}^{*}(t, x) & =j_{\sigma}^{\prime}\left(u_{\varepsilon, \sigma}^{*}(t, x)\right), & \text { a.e. }(t, x) & \in Q,
\end{aligned}
$$

and $p_{\varepsilon, \sigma}^{*}$ is the solution to

$$
\begin{aligned}
\left(p_{\varepsilon, \sigma}\right)_{t}+\Delta p_{\varepsilon, \sigma} & =-\lambda_{1}\left(y_{\varepsilon, \sigma}^{*}-w_{\varepsilon, \sigma}^{*}\right), & & \text { in } Q, \\
\frac{\partial p_{\varepsilon, \sigma}}{\partial \nu} & =0, & & \text { on } \Sigma, \\
p_{\varepsilon, \sigma}(T) & =0, & & \text { in } \Omega .
\end{aligned}
$$

Proof. Let $\lambda \in[0,1]$ and write $w_{\varepsilon, \sigma}^{\lambda}=w_{\varepsilon, \sigma}^{*}+\lambda W, W=w-w_{\varepsilon, \sigma}^{*}$, for any $w \in L^{\infty}(Q), w(t, x) \in K$ a.e. $(t, x) \in Q$. We write that $v_{\varepsilon, \sigma}^{*}, w_{\varepsilon, \sigma}^{*}$ is optimal in (3.8), that is

$$
J_{\varepsilon, \sigma}\left(v_{\varepsilon, \sigma}^{*}, w_{\varepsilon, \sigma}^{*}\right) \leq J_{\varepsilon, \sigma}(\widehat{v}, \widehat{w}), \text { for all } \widehat{v} \in L^{2}(Q), \widehat{w}(t, x) \in[b, c], \text { a.e. }(t, x) \in Q,
$$

and set $\widehat{v}=v_{\varepsilon, \sigma}^{*}$ and $\widehat{w}=w_{\varepsilon, \sigma}^{\lambda}$. We obtain after a few calculations

$$
\int_{Q}\left(-\lambda_{1}\left(y_{\varepsilon, \sigma}^{*}-w_{\varepsilon, \sigma}^{*}\right)-\frac{1}{\varepsilon} u_{\varepsilon, \sigma}^{*}+w_{\varepsilon, \sigma}^{*}-w_{\varepsilon}^{*}\right) W d x d t \geq 0,
$$

whence we get

$$
\int_{Q}\left(w_{\varepsilon, \sigma}^{*}-w\right)\left(\lambda_{1}\left(y_{\varepsilon, \sigma}^{*}-w_{\varepsilon, \sigma}^{*}\right)+\frac{1}{\varepsilon} u_{\varepsilon, \sigma}^{*}-w_{\varepsilon, \sigma}^{*}+w_{\varepsilon}^{*}\right) d x d t \geq 0,
$$

for all $w \in L^{\infty}(Q), w(t, x) \in[b, c]$ a.e. $(t, x) \in Q$. This yields

$$
w_{\varepsilon, \sigma}^{*}+\frac{1}{1+\lambda_{1}} \partial I_{K}\left(w_{\varepsilon, \sigma}^{*}\right) \ni \frac{1}{1+\lambda_{1}}\left(\lambda_{1} y_{\varepsilon, \sigma}^{*}+\frac{1}{\varepsilon} u_{\varepsilon, \sigma}^{*}+w_{\varepsilon}^{*}\right),
$$

which still reads

$$
w_{\varepsilon, \sigma}^{*}=P_{K}\left(\frac{1}{1+\lambda_{1}}\left(\lambda_{1} y_{\varepsilon, \sigma}^{*}+\frac{1}{\varepsilon} u_{\varepsilon, \sigma}^{*}+w_{\varepsilon}^{*}\right)\right),
$$

where $P_{K}(z)$ is the projection of the point $z$ on the set $K$. From here we get (4.1).

Now, let $\lambda>0$ and consider the variation $v_{\varepsilon, \sigma}^{\lambda}=v_{\varepsilon, \sigma}^{*}+\lambda V$, for all $V \in L^{2}(Q)$. If we denote by $y_{\varepsilon, \sigma}^{\lambda}$ and $u_{\varepsilon, \sigma}^{\lambda}$ the solutions to (1.12)-(1.13) corresponding to $v=v_{\varepsilon, \sigma}^{\lambda}$, by $U$ the solution to (1.13) corresponding to $v=V$ and by $Y$ the weak limit in $L^{2}(Q)$ defined as

$$
Y(t, x)=\lim _{\lambda \rightarrow 0} \frac{y_{\varepsilon, \sigma}^{v_{\varepsilon}^{\lambda}}(t, x)-y^{v_{\varepsilon, \sigma}^{*}}(t, x)}{\lambda},
$$

we get by a direct calculation that $Y$ and $U$ satisfy the systems in variations

$$
\begin{aligned}
Y_{t}-\Delta Y & =U, \text { in } Q, \\
-\frac{\partial Y}{\partial \nu} & =0, \text { on } \Sigma, \\
Y(0) & =0, \text { in } \Omega,
\end{aligned}
$$




$$
\begin{aligned}
U_{t}-\Delta U & =V, \text { in } Q, \\
U & =0, \quad \text { on } \Sigma, \\
U(0) & =0, \text { in } \Omega .
\end{aligned}
$$

Obviously, each of these systems has a unique solution

$$
\begin{aligned}
& U \in C\left([0, T] ; H_{0}^{1}(\Omega)\right] \cap W^{1,2}\left([0, T] ; L^{2}(\Omega)\right) \cap L^{2}\left(0, T ; H^{2}(\Omega)\right), \\
& Y \in C\left([0, T] ; H^{1}(\Omega)\right) \cap W^{1,2}\left([0, T] ; L^{2}(\Omega)\right) \cap L^{2}\left(0, T ; H^{2}(\Omega)\right) .
\end{aligned}
$$

We introduce the dual systems (4.6),

$$
\begin{aligned}
\left(p_{\varepsilon, \sigma}\right)_{t}+\Delta p_{\varepsilon, \sigma} & =-\lambda_{1}\left(y_{\varepsilon, \sigma}^{*}-w_{\varepsilon, \sigma}^{*}\right), & & \text { in } Q, \\
\frac{\partial p_{\varepsilon, \sigma}}{\partial \nu} & =0, & & \text { on } \Sigma, \\
p_{\varepsilon, \sigma}(T) & =0, & & \text { in } \Omega,
\end{aligned}
$$

and

$$
\begin{aligned}
\left(q_{\varepsilon, \sigma}\right)_{t}+\Delta q_{\varepsilon, \sigma} & =-\frac{1}{\varepsilon}\left(\xi_{\varepsilon, \sigma}^{*}-w_{\varepsilon}^{*}\right), & & \text { in } Q, \\
q_{\varepsilon, \sigma} & =0, & & \text { on } \Sigma, \\
q_{\varepsilon, \sigma}(T) & =0, & & \text { in } \Omega,
\end{aligned}
$$

where

$$
\xi_{\varepsilon, \sigma}^{*}=j_{\sigma}^{\prime}\left(u_{\varepsilon, \sigma}^{*}\right)
$$

Systems (4.6) and (4.11) have a unique solution

$$
\begin{aligned}
& p_{\varepsilon, \sigma} \in C\left([0, T] ; H^{1}(\Omega)\right] \cap W^{1,2}\left([0, T] ; L^{2}(\Omega)\right) \cap L^{2}\left(0, T ; H^{2}(\Omega)\right), \\
& q_{\varepsilon, \sigma} \in C\left([0, T] ; H_{0}^{1}(\Omega)\right] \cap W^{1,2}\left([0, T] ; L^{2}(\Omega)\right) \cap L^{2}\left(0, T ; H^{2}(\Omega)\right) .
\end{aligned}
$$

We write again that $w_{\varepsilon, \sigma}^{*}, v_{\varepsilon, \sigma}^{*}$ is optimal in (3.8),

$$
J_{\varepsilon, \sigma}\left(v_{\varepsilon, \sigma}^{*}, w_{\varepsilon, \sigma}^{*}\right) \leq J_{\varepsilon, \sigma}(\widehat{v}, \widehat{w}) \text {, for all } \widehat{v} \in L^{2}(Q), \widehat{w}(t, x) \in[b, c] \text { a.e. }(t, x) \in Q .
$$

In particular, we set $\widehat{v}=v_{\varepsilon, \sigma}^{\lambda}, \widehat{w}=w_{\varepsilon, \sigma}^{*}$ and after dividing by $\lambda$ and letting it to go to 0 , we get

$$
\int_{Q} \lambda_{1}\left(y_{\varepsilon, \sigma}^{*}-w_{\varepsilon, \sigma}^{*}\right) Y d x d t+\int_{Q}\left(\left(\lambda_{2}+1\right) v_{\varepsilon, \sigma}^{*}-v_{\varepsilon}^{*}\right) V d x d t+\frac{1}{\varepsilon} \int_{Q}\left(\xi_{\varepsilon, \sigma}^{*}-w_{\varepsilon, \sigma}^{*}\right) U d x d t \geq 0,
$$

for all $V \in L^{2}(Q)$. Changing $\lambda$ into $(-\lambda)$ we finally obtain

$$
\int_{Q} \lambda_{1}\left(y_{\varepsilon, \sigma}^{*}-w_{\varepsilon, \sigma}^{*}\right) Y d x d t+\int_{Q}\left(\left(\lambda_{2}+1\right) v_{\varepsilon, \sigma}^{*}-v_{\varepsilon}^{*}\right) V d x d t+\frac{1}{\varepsilon} \int_{Q}\left(\xi_{\varepsilon, \sigma}^{*}-w_{\varepsilon, \sigma}^{*}\right) U d x d t=0,
$$

for all $V \in L^{2}(Q)$.

Next, we multiply (4.9) by $p_{\varepsilon, \sigma}$, add with (4.10) multiplied by $q_{\varepsilon, \sigma}$, and integrate over $Q$. We obtain

$$
-\int_{Q}\left(\left(p_{\varepsilon, \sigma}\right)_{t}+\Delta p_{\varepsilon, \sigma}\right) Y d x d t-\int_{Q}\left(\left(q_{\varepsilon, \sigma}\right)_{t}+\Delta q_{\varepsilon, \sigma}\right) U d x d t=\int_{Q}\left(U p_{\varepsilon, \sigma}+V q_{\varepsilon, \sigma}\right) d x d t
$$

and further

$$
\int_{Q} \lambda_{1}\left(y_{\varepsilon, \sigma}^{*}-w_{\varepsilon, \sigma}^{*}\right) Y d x d t+\frac{1}{\varepsilon} \int_{Q}\left(\xi_{\varepsilon, \sigma}^{*}-w_{\varepsilon, \sigma}^{*}\right) U d x d t=\int_{Q}\left(U p_{\varepsilon, \sigma}+V q_{\varepsilon, \sigma}\right) d x d t .
$$


Plugging this relation in (4.12) we get

$$
\int_{Q} U p_{\varepsilon, \sigma} d x d t+\int_{Q}\left(q_{\varepsilon, \sigma}+\lambda_{2} v_{\varepsilon, \sigma}^{*}+v_{\varepsilon, \sigma}^{*}-v_{\varepsilon}^{*}\right) V d x d t=0, \text { for all } V \in L^{2}(Q) .
$$

By (4.10) we write the solution representation

$$
U(t)=\int_{0}^{t} e^{\Delta(t-s)} V(s) d s, t \geq 0,
$$

where $e^{\Delta t}$ is the semigroup generated by the operator Laplace with Dirichlet boundary conditions, and replace it in (4.14), obtaining

$$
\int_{Q}\left(q_{\varepsilon, \sigma}+\left(\lambda_{2}+1\right) v_{\varepsilon, \sigma}^{*}-v_{\varepsilon}^{*}\right) V d x d t+\int_{0}^{T} \int_{\Omega} p_{\varepsilon, \sigma}(t) e^{\Delta t} \int_{0}^{t} e^{-\Delta s} V(s) d s d x d t=0 .
$$

Changing the order of integrals in the second term we finally deduce

$$
\int_{0}^{T} \int_{\Omega} V(t)\left(\int_{t}^{T} e^{-\Delta(t-s)} p_{\varepsilon, \sigma}(s) d s+q_{\varepsilon, \sigma}+\left(\lambda_{2}+1\right) v_{\varepsilon, \sigma}^{*}-v_{\varepsilon}^{*}\right) d x d t=0,
$$

for all $V \in L^{2}(Q)$, which implies

$$
\left(1+\lambda_{2}\right) v_{\varepsilon, \sigma}^{*}-v_{\varepsilon}^{*}+q_{\varepsilon, \sigma}=-\int_{t}^{T} e^{-\Delta(t-s)} p_{\varepsilon, \sigma}(s) d s .
$$

Actually, denoting $z_{\varepsilon, \sigma}^{*}=\left(1+\lambda_{2}\right) v_{\varepsilon, \sigma}^{*}-v_{\varepsilon}^{*}$ we have that $z_{\varepsilon, \sigma}^{*}$ is provided by the equation

$$
\begin{array}{rlrl}
\left(z_{\varepsilon, \sigma}^{*}\right)_{t}+\left(q_{\varepsilon, \sigma}\right)_{t}+\Delta z_{\varepsilon, \sigma}^{*}+\Delta q_{\varepsilon, \sigma} & =p_{\varepsilon, \sigma}, & \text { in } Q, \\
z_{\varepsilon, \sigma}^{*}+q_{\varepsilon, \sigma} & =0, & & \text { on } \Sigma, \\
\left(z_{\varepsilon, \sigma}^{*}+q_{\varepsilon, \sigma}\right)(T) & =0, & & \text { in } \Omega,
\end{array}
$$

whence taking into account (4.11) we get (4.4).

Problem (4.4) has a unique solution

$$
z_{\varepsilon, \sigma}^{*}=\left(1+\lambda_{2}\right) v_{\varepsilon, \sigma}^{*}-v_{\varepsilon}^{*} \in C\left([0, T] ; H_{0}^{1}(\Omega)\right] \cap W^{1,2}\left([0, T] ; L^{2}(\Omega)\right) \cap L^{2}\left(0, T ; H^{2}(\Omega)\right) .
$$

In conclusion, recalling that $\left\{v_{\varepsilon, \sigma}^{*}, w_{\varepsilon, \sigma}^{*}\right\}$ denotes an optimal controller in (3.8), with $\left\{u_{\varepsilon, \sigma}^{*}, y_{\varepsilon, \sigma}^{*}\right\}$ the corresponding states, we get (4.1)-(4.6) as claimed.

Finally, as proved in Theorem 3.2, each solution $v_{\varepsilon}^{*}, w_{\varepsilon}^{*}$ to $(3.3)$ is the strong limit of the sequences $\left\{v_{\varepsilon, \sigma}^{*}\right\}_{\varepsilon>0}$ and $\left\{w_{\varepsilon, \sigma}^{*}\right\}_{\varepsilon>0}$ having the expressions given by (4.1) and (4.2), so that we can conclude with the next Proposition.

Proposition 4.2. Let $y_{0} \in L^{2}(\Omega), u_{0} \in L^{2}(\Omega), f \in L^{2}(Q), g \in L^{2}(\Sigma)$ and let $\left\{v_{\varepsilon}^{*}, w_{\varepsilon}^{*}\right\}$ be an optimal controller in (3.3). Then, the optimality conditions for (3.3) read as

$$
w_{\varepsilon}^{*}(t, x)= \begin{cases}b, & \text { in }\left\{(t, x) ; a_{\varepsilon}^{*}(t, x) \leq b\right\} \\ a_{\varepsilon}^{*}(t, x), & \text { in }\left\{(t, x) ; b<a_{\varepsilon}^{*}(t, x)<c\right\} \\ c, & \text { in }\left\{(t, x) ; a_{\varepsilon}^{*}(t, x) \geq c\right\}\end{cases}
$$

where

$$
a_{\varepsilon}^{*}(t, x)=y_{\varepsilon}^{*}(t, x)+\frac{1}{\varepsilon \lambda_{1}} u_{\varepsilon}^{*}(t, x)
$$


and

$$
v_{\varepsilon}^{*}(t, x)=\frac{z_{\varepsilon}^{*}(t, x)}{\lambda_{2}}
$$

where $z_{\varepsilon}^{*}$ is a solution to the problem

$$
\begin{aligned}
\left(z_{\varepsilon}^{*}\right)_{t}+\Delta z_{\varepsilon}^{*} & \in p_{\varepsilon}+\frac{1}{\varepsilon}\left(H_{b, c}\left(u_{\varepsilon}^{*}\right)-w_{\varepsilon}^{*}\right), & & \text { in } Q, \\
z_{\varepsilon}^{*} & =0, & & \text { on } \Sigma, \\
\left(z_{\varepsilon}^{*}\right)(T) & =0, & & \text { in } \Omega,
\end{aligned}
$$

and $p_{\varepsilon}$ is the unique solution to the problem

$$
\begin{aligned}
\left(p_{\varepsilon}\right)_{t}+\Delta p_{\varepsilon} & =-\lambda_{1}\left(y_{\varepsilon}^{*}-w_{\varepsilon}^{*}\right), & & \text { in } Q, \\
\frac{\partial p_{\varepsilon}}{\partial \nu} & =0, & & \text { on } \Sigma, \\
p_{\varepsilon}(T) & =0, & & \text { in } \Omega .
\end{aligned}
$$

Proof. By Theorem 3.2, we have (3.9) and that

$$
u_{\varepsilon, \sigma}^{*} \rightarrow u_{\varepsilon}^{*}, \quad y_{\varepsilon, \sigma}^{*} \rightarrow y_{\varepsilon}^{*} \text { strongly in } L^{2}(Q), \text { as } \sigma \rightarrow 0 .
$$

Then, we pass to the limit in (4.1) and deduce

$$
w_{\varepsilon}^{*}(t, x)= \begin{cases}b, & \text { on }\left\{(t, x) ; a_{\varepsilon}^{*}(t, x)=b\right\}, \\ a_{\varepsilon}^{*}(t, x), & \text { on }\left\{(t, x) ; b<a_{\varepsilon}^{*}(t, x)<c\right\}, \\ c, & \text { on }\left\{(t, x) ; a_{\varepsilon}^{*}(t, x)=c\right\},\end{cases}
$$

where $a_{\varepsilon}^{*}(t, x)=\frac{1}{1+\lambda_{1}}\left(\lambda_{1} y_{\varepsilon}^{*}+\frac{1}{\varepsilon} u_{\varepsilon}^{*}+w_{\varepsilon}^{*}\right)(t, x)$. After a few algebra this implies (4.19). We note that

$$
\xi_{\varepsilon, \sigma}^{*} \rightarrow \xi_{\varepsilon}^{*} \text { weak }{ }^{*} \text { in } L^{\infty}(Q), \xi_{\varepsilon}^{*}(t, x) \in \partial j\left(u_{\varepsilon}^{*}(t, x)\right) \text { a.e. }(t, x) \in Q .
$$

Indeed,

$$
\int_{Q}\left(j_{\sigma}\left(u_{\varepsilon, \sigma}^{*}\right)-j_{\sigma}(z)\right) d x d t \leq \int_{Q} j_{\sigma}^{\prime}\left(u_{\varepsilon, \sigma}^{*}\right)\left(u_{\varepsilon, \sigma}^{*}-z\right) d x d t
$$

for any $z \in L^{2}(Q)$. Passing to the limit as $\sigma \rightarrow 0$ and recalling (3.13) and (3.7) we get

$$
\int_{Q}\left(j\left(u_{\varepsilon}^{*}\right)-j(z)\right) d x d t \leq \int_{Q} \xi_{\varepsilon}^{*}\left(u_{\varepsilon}^{*}-z\right) d x d t
$$

whence $\xi_{\varepsilon}^{*}(t, x) \in \partial j\left(u_{\varepsilon}^{*}(t, x)\right)$ a.e. $(t, x) \in Q$.

By (4.6) we get that

$$
\begin{aligned}
p_{\varepsilon, \sigma} \rightarrow p_{\varepsilon} & \text { weakly in } W^{1,2}\left([0, T] ; L^{2}(\Omega)\right) \cap L^{2}\left(0, T ; H^{2}(\Omega)\right), \\
& \text { weak* in } L^{\infty}\left(0, T ; H^{1}(\Omega)\right), \\
& \text { strongly in } L^{2}\left(0, T ; H^{1}(\Omega)\right), \text { as } \sigma \rightarrow 0
\end{aligned}
$$

and so, $p_{\varepsilon}$ satisfies (4.23).

By (4.4) we have,

$$
\begin{aligned}
z_{\varepsilon, \sigma}^{*} \rightarrow z_{\varepsilon}^{*} & \text { weakly in } W^{1,2}\left([0, T] ; L^{2}(\Omega)\right) \cap L^{2}\left(0, T ; H^{2}(\Omega)\right), \\
& \text { weak }^{*} \text { in } L^{\infty}\left(0, T ; H_{0}^{1}(\Omega)\right), \\
& \text { strongly in } L^{2}\left(0, T ; H_{0}^{1}(\Omega)\right), \text { as } \sigma \rightarrow 0,
\end{aligned}
$$


and

$$
z_{\varepsilon, \sigma}^{*}(t) \rightarrow z_{\varepsilon}^{*}(t) \text { strongly in } L^{2}(\Omega), \text { uniformly in } t \text { on compacts, }
$$

by the Arzelà-Ascoli theorem. Moreover, $z_{\varepsilon}^{*}$ satisfies the equation

$$
\begin{aligned}
\left(z_{\varepsilon}^{*}\right)_{t}+\Delta z_{\varepsilon}^{*} & =p_{\varepsilon}+\frac{1}{\varepsilon}\left(\xi_{\varepsilon}^{*}-w_{\varepsilon}^{*}\right), & & \text { in } Q, \\
z_{\varepsilon}^{*} & =0, & & \text { on } \Sigma, \\
\left(z_{\varepsilon}^{*}\right)(T) & =0, & & \text { in } \Omega,
\end{aligned}
$$

where $\xi_{\varepsilon}^{*}(t, x) \in \partial j\left(u_{\varepsilon}^{*}(t, x)\right)$ a.e. $(t, x) \in Q$. Then, by (4.2) we get (4.21).

\section{Numerical results}

In the last section we present some numerical simulations based on an algorithm developed for computing the approximating solution to (3.3). For simplicity we shall not write the subscript $\varepsilon$ for $v_{\varepsilon}^{*}, w_{\varepsilon}^{*}$ and the other functions.

Fix $\varepsilon_{\text {stop }}$ and indicate the problem data.

Give $y_{0}(x), u_{0}(x)$.

Step 0. Choose the "0"-order values for the controls $w_{0}^{*}$ and $v_{0}^{*}$. Set $k:=0$.

Step $0_{1}$. Determine $u_{k}^{*}$ by (1.13) with $v=v_{k}^{*}$ (here $v_{0}^{*}$ ),

$$
\begin{aligned}
\left(u_{k}^{*}\right)_{t}-\Delta u_{k}^{*} & =v_{k}^{*}, \text { in } Q, \\
u_{k}^{*} & =0, \text { on } \Sigma, \\
u_{k}^{*}(0) & =u_{0}, \text { in } \Omega .
\end{aligned}
$$

Determine $y_{k}^{*}$ by (1.12), with $u=u_{k}^{*}$,

$$
\begin{aligned}
\left(y_{k}^{*}\right)_{t}-\Delta y_{k}^{*} & =u_{k}^{*}+f, \text { in } Q, \\
-\frac{\partial y_{k}^{*}}{\partial \nu} & =g, \text { on } \Sigma, \\
y_{k}^{*}(0) & =y_{0}, \text { in } \Omega,
\end{aligned}
$$

and $J_{k}\left(v_{k}^{*}, w_{k}^{*}\right)$ by $(3.2)$,

$$
J_{k}\left(v_{k}^{*}, w_{k}^{*}\right)=\frac{\lambda_{1}}{2} \int_{Q}\left(y_{k}^{*}-w_{k}^{*}\right)^{2} d x d t+\frac{\lambda_{2}}{2} \int_{Q}\left(v_{k}^{*}\right)^{2} d x d t+\frac{1}{\varepsilon} \int_{Q}\left(j\left(u_{k}^{*}\right)-u_{k}^{*} w_{k}^{*}\right) d x d t .
$$

Step $\mathrm{O}_{2}$. Compute (for using in the next step) the following: $a_{k}^{*}$ by (4.20),

$$
a_{k}^{*}=y_{k}^{*}+\frac{1}{\varepsilon \lambda_{1}} u_{k}^{*}
$$

$p_{k}$ by (4.23),

$$
\begin{aligned}
\left(p_{k}\right)_{t}+\Delta p_{k} & =-\lambda_{1}\left(y_{k}^{*}-w_{k}^{*}\right), & & \text { in } Q, \\
\frac{\partial p_{k}}{\partial \nu} & =0, & & \text { on } \Sigma, \\
p_{k}(T) & =0, & & \text { in } \Omega,
\end{aligned}
$$


$z_{k}^{*}$ by $(4.22)$,

$$
\begin{aligned}
\left(z_{k}^{*}\right)_{t}+\Delta z_{k}^{*} & \in p_{k}+\frac{1}{\varepsilon}\left(\xi_{\varepsilon}^{*}-w_{k}^{*}\right), & & \text { in } Q, \\
z_{k}^{*} & =0, & & \text { on } \Sigma, \\
\left(z_{k}^{*}\right)(T) & =0, & & \text { in } \Omega, \\
\xi_{\varepsilon}^{*}(t, x) & \in H_{b, c}\left(u_{k}^{*}(t, x)\right) & \text { a.e. } & (t, x) \in Q .
\end{aligned}
$$

Finally, compute the function given by (4.19), which here is denoted

$$
W_{k}(t, x)= \begin{cases}b, & \text { in }\left\{(t, x) ; a_{k}^{*}(t, x) \leq b\right\}, \\ a_{k}^{*}(t, x), & \text { in }\left\{(t, x) ; b<a_{k}^{*}(t, x)<c\right\}, \\ c, & \text { in }\left\{(t, x) ; a_{k}^{*}(t, x) \geq c\right\},\end{cases}
$$

and the function given by (4.21), denoted

$$
V_{k}(t, x)=\frac{z_{k}^{*}(t, x)}{\lambda_{2}}
$$

This ends Step 0.

Step $k+1$. Make $k:=k+1$.

Step $k_{1}$. Following Rosen algorithm (see [1], p. 44) compute $\lambda$ which realizes

$$
\min \left\{J_{k+1}\left(v_{k+1}^{\lambda}, w_{k+1}^{\lambda}\right) ; \lambda \in(0,1]\right\},
$$

where the variations $w_{k+1}^{\lambda}, v_{k+1}^{\lambda}$ are determined using the functions $W_{k}$ and $V_{k}$, i.e.,

$$
\begin{gathered}
w_{k+1}^{\lambda}(t, x)=w_{k}^{*}(t, x)+\lambda W_{k}(t, x), \lambda \in(0,1], \\
v_{k+1}^{\lambda}(t, x)=v_{k}^{*}(t, x)+\lambda V_{k}(t, x) .
\end{gathered}
$$

In the expression of $J_{k+1}, u_{k+1}^{\lambda}$ is given by (1.13) with $v=v_{k+1}^{\lambda}$ and $y_{k+1}^{\lambda}$ is determined by (1.12) with $u=u_{k+1}^{\lambda}$.

In order to achieve (5.1) we make the following remark. Step $k_{1}$ is run for a sequence of $p$ values $\lambda_{p}=p * \operatorname{step}_{\lambda}, \operatorname{step}_{\lambda} \in(0,1), p=1, \ldots, 1 / \operatorname{step}_{\lambda}$.

Step $k_{2}$. Pick up that $\lambda_{p}$ which realizes the minimum in (5.1), denote it $\lambda_{\text {min }}$, set

$$
J_{k+1}\left(v_{k+1}^{\lambda_{\min }}, w_{k+1}^{\lambda_{\min }}\right)=\min \left\{J_{k+1}\left(v_{k+1}^{\lambda}, w_{k+1}^{\lambda}\right) ; \lambda \in(0,1]\right\},
$$

and then set

Step $k_{3}$. Denote

$$
\begin{gathered}
v_{k+1}^{*}(t, x)=v_{k+1}^{\lambda_{\min }}(t, x), w_{k+1}^{*}(t, x)=w_{k+1}^{\lambda_{\min }}(t, x), \\
u_{k+1}^{*}(t, x)=u_{k+1}^{\lambda_{\min }}(t, x), y_{k+1}^{*}(t, x)=y_{k+1}^{\lambda_{\min }}(t, x), \\
J_{k+1}\left(v_{k+1}^{*}, w_{k+1}^{*}\right)=J_{k+1}\left(v_{k+1}^{\lambda_{\min }}, y_{k+1}^{\lambda_{\min }}\right) .
\end{gathered}
$$

$$
\operatorname{err}_{k+1}=J_{k+1}\left(v_{k+1}^{*}, w_{k+1}^{*}\right)-J_{k}\left(v_{k}^{*}, w_{k}^{*}\right) .
$$

If $\left|\operatorname{err}_{k+1}\right| \leq \varepsilon_{\text {stop }}$, then the algorithm stops and set $\left\{v_{k+1}^{*}, w_{k+1}^{*}\right\}$ and $\left\{u_{k+1}^{*}, y_{k+1}^{*}\right\}$ as optimal. If not, go to the next step.

Step $k_{4}$. Compute (for using in a possible next step) $a_{k+1}^{*}$ by (4.20), $W_{k+1}$ by (4.19), $p_{k+1}$ by (4.23), $z_{k+1}^{*}$ by $(4.22), V_{k+1}$ by $(4.21)$ and continue from Step $k_{1}$.

The 1D numerical simulations have been obtained with the software Mathematica (license L4698-0010), for the following data:

$$
\begin{aligned}
\Omega & =[0, L], L=2, T=1, \lambda_{1}=1, \lambda_{2}=0.1, \\
b & =0.1, c=1, g=0, \varepsilon=0.1, \\
\text { step }_{\lambda} & =0.001, p=10, \varepsilon_{\text {stop }}=0.1 .
\end{aligned}
$$


The computation of $\xi_{\varepsilon}^{*}$ was done by approximating it by $\xi_{\varepsilon, \sigma}^{*}$ (see (4.5)) with $\sigma=\varepsilon_{H}=0.00001$.

Case 1. We considered:

$$
y_{0}(x)=0.9 x^{2}(L-x)^{2}, u_{0}(x)=0, w_{0}^{*}(x)=0.5, v_{0}^{*}(x)=1, f=0 .
$$

The surfaces $z=y_{k}^{*}(t, x)$ and $z=w_{k}^{*}(t, x)$ obtained after $k=9$ steps are represented in Figure 1.1 and Figure 1.2.

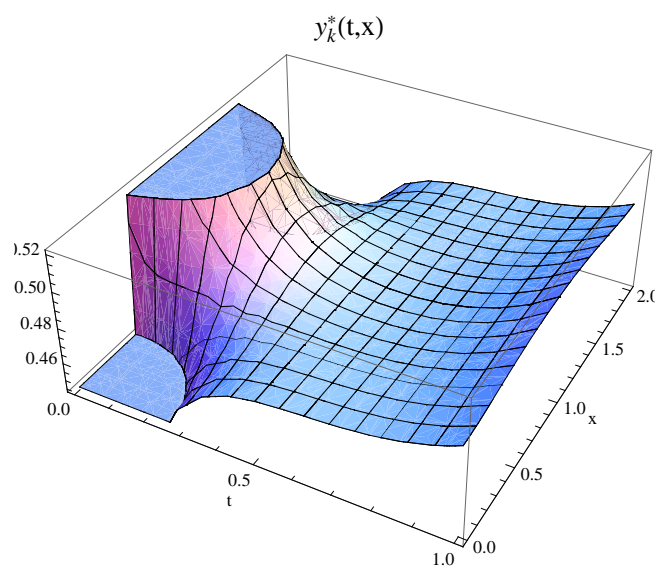

FIGURE 1.1: Case 1. $y_{k}^{*}(t, x), k=9$

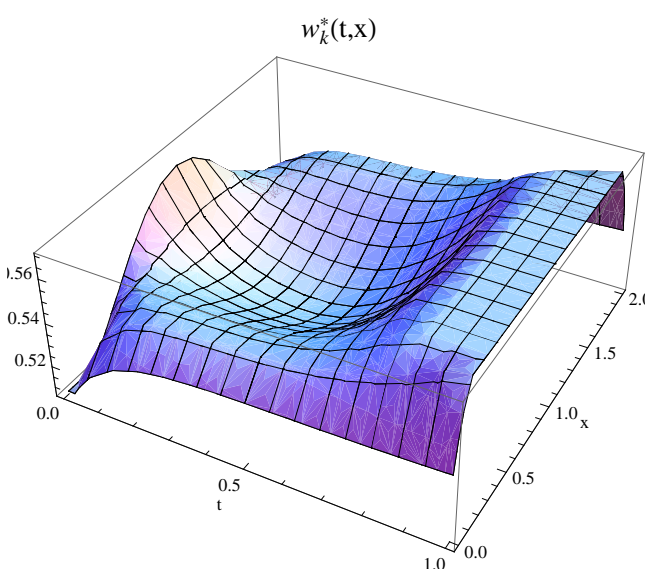

FIGURE 1.2: Case 1. $w_{k}^{*}(t, x), k=9$

The contour plots of $y_{k}^{*}(t, x)$ and $w_{k}^{*}(t, x)$ (after 9 steps) are plotted in Figure 1.3 and Figure 1.4, for $t \in[0, T]$. One can observe there the evolution of the level curves from $t=0$ to $t=T$.

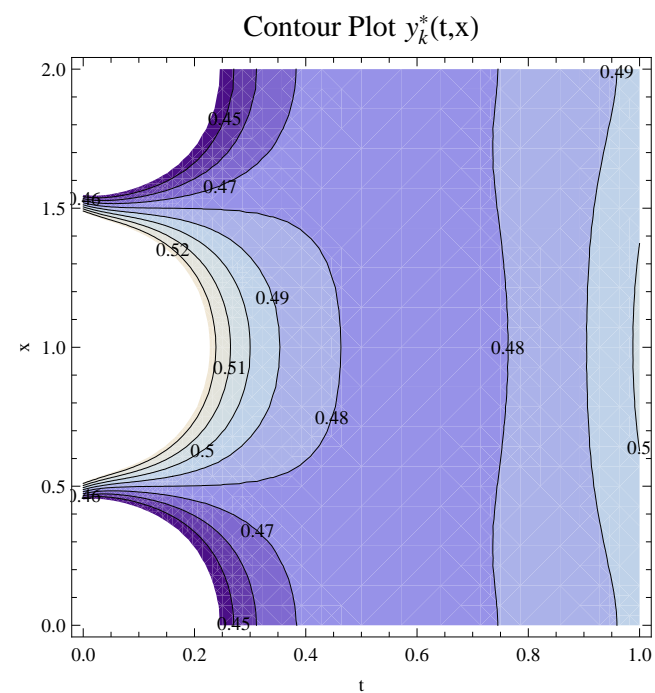

FIGURE 1.3: Case 1. $y_{k}^{*}(t, x), t \in[0, T]$

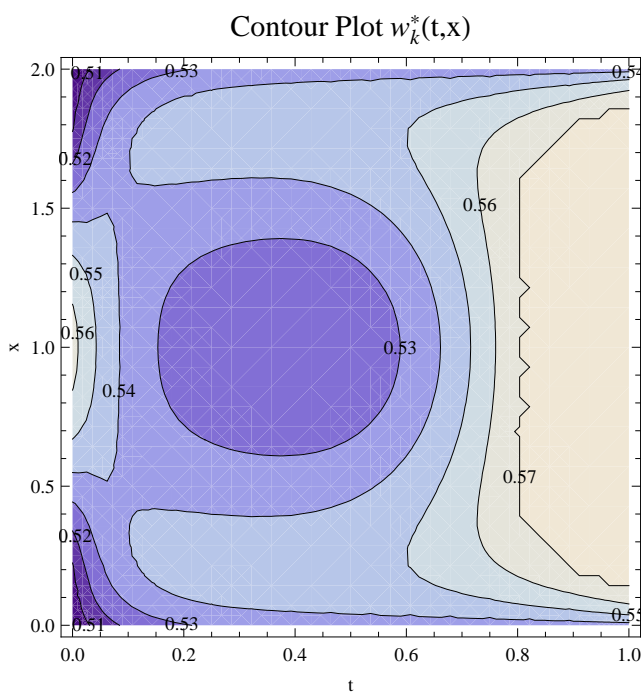

FIGURE 1.4: Case 1. $w_{k}^{*}(t, x), t \in[0, T]$

The contour plots of $y_{k}^{*}(t, x)$ and $w_{k}^{*}(t, x)$ for $t \in[0, T / 10]$ are given in Figure 1.5 and Figure 1.6. These figures make a zoom in the time interval $[0,0.1]$ and put into evidence the regions $Q_{b}$ in the left corners of Figures 1.5 and 1.6. Figures $1.3-1.6$ show the evolution of the regions $Q_{b, c}(t), Q_{b}(t)\left(Q_{c}(t)\right.$ being not formed). 


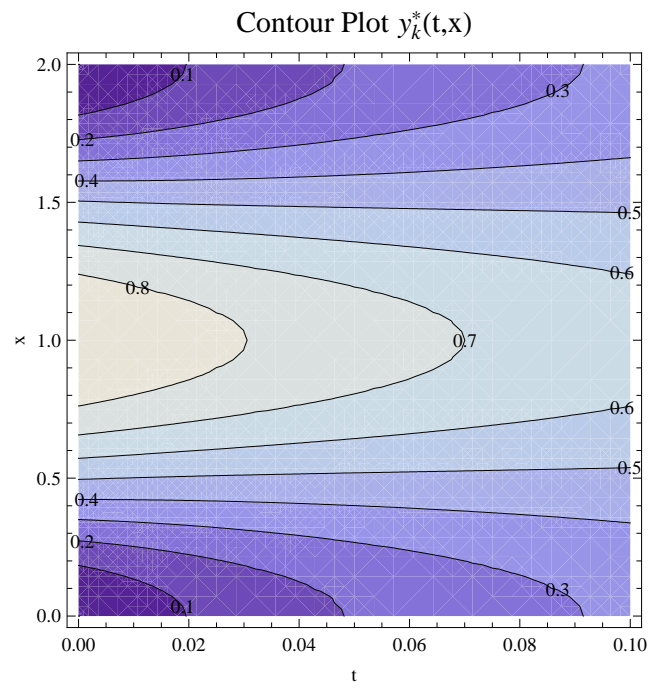

FIGURE 1.5: Case 1. $y_{k}^{*}(t, x), t \in[0, T / 10]$

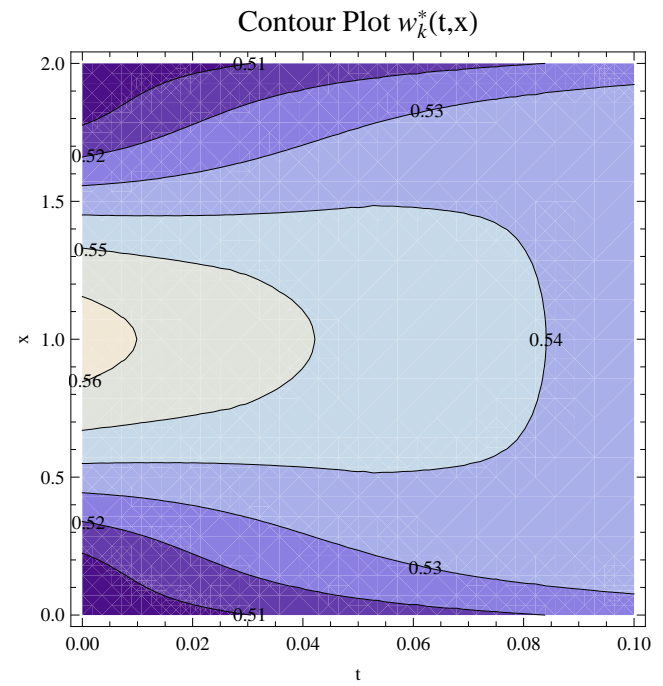

FIGURE 1.6: Case 1. $w_{k}^{*}(t, x), t \in[0, T / 10]$

The values of $J_{k}\left(v_{k}^{*}, w_{k}^{*}\right)$ for $k=0, \ldots, 9$ are : $15.5428,14.9529,14.3786,13.8208,13.2773,12.7492$, $12.2395,11.9325,11.8396,11.8308$.

Case 2 presents in Figures $2.1-2.6$ similar graphics obtained after $k=8$ steps for

$$
y_{0}(x)=0.5, u_{0}(x)=0, w_{0}^{*}(x)=0.5, v_{0}^{*}(x)=1, f=0 .
$$

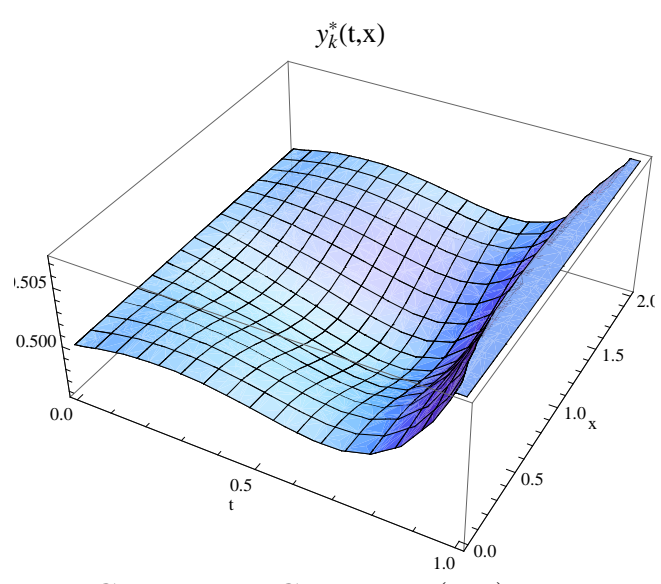

FIGURE 2.1: Case 2. $y_{k}^{*}(t, x), k=8$

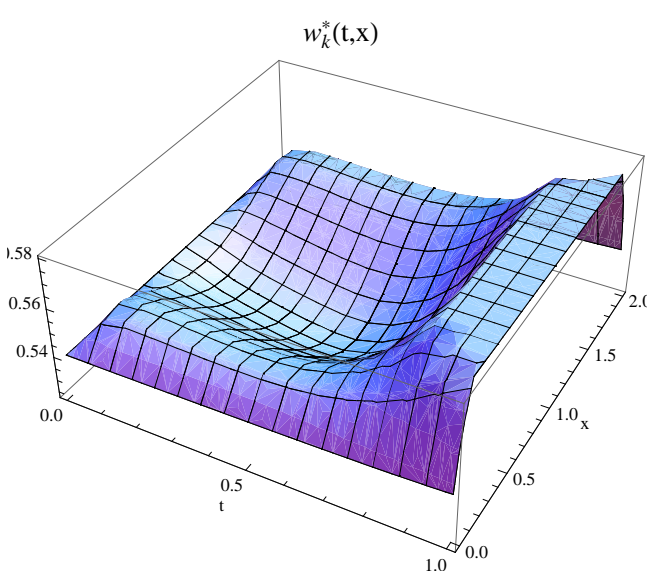

FIGURE 2.2: Case 2. $w_{k}^{*}(t, x), k=8$ 


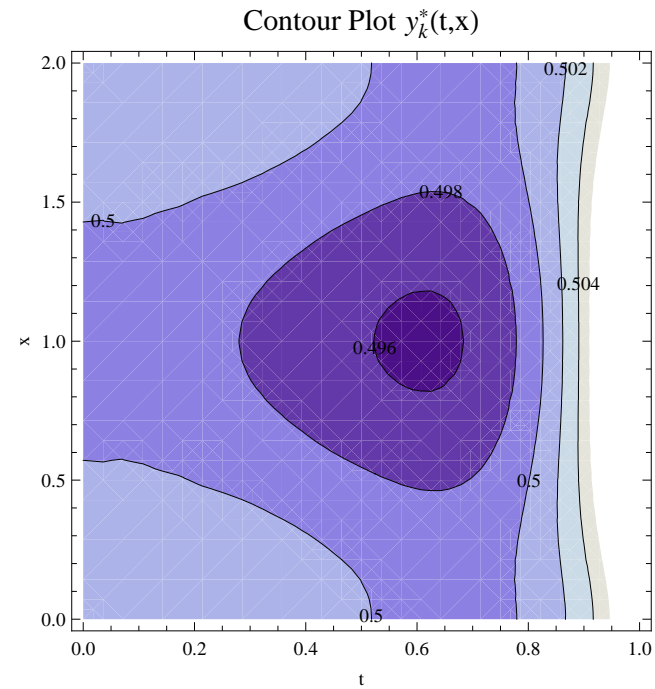

FIGURE 2.3: Case 2. $y_{k}^{*}(t, x), t \in[0, T]$

Contour Plot $y_{k}^{*}(\mathrm{t}, \mathrm{x})$

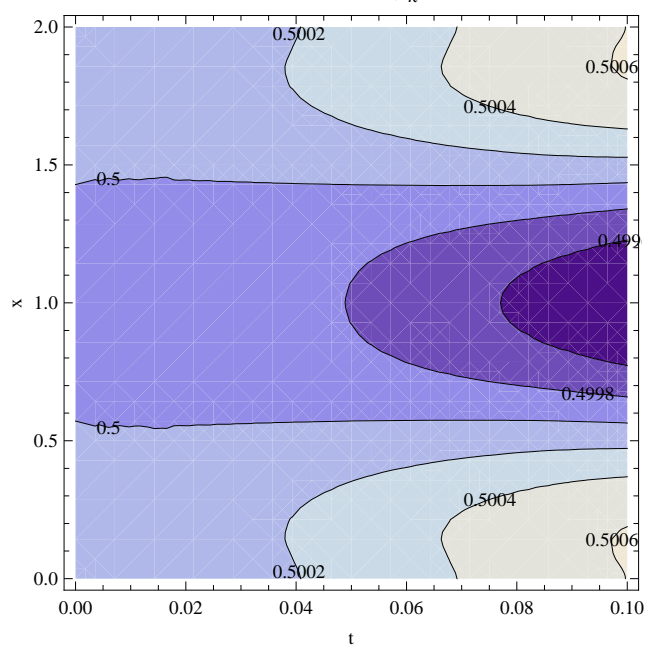

FIGURE 2.5: Case 2. $y_{k}^{*}(t, x), t \in[0, T / 10]$

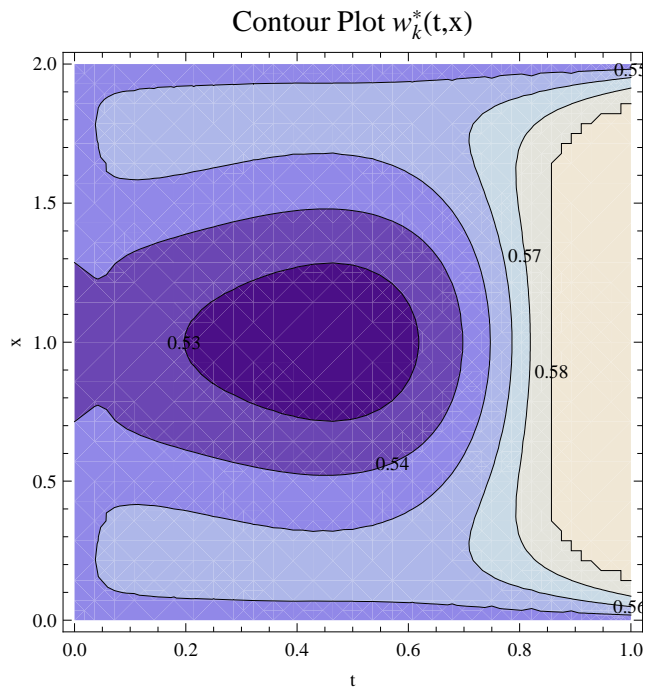

FIGURE 2.4: Case 2. $w_{k}^{*}(t, x), t \in[0, T]$

Contour Plot $w_{k}^{*}(\mathrm{t}, \mathrm{x})$

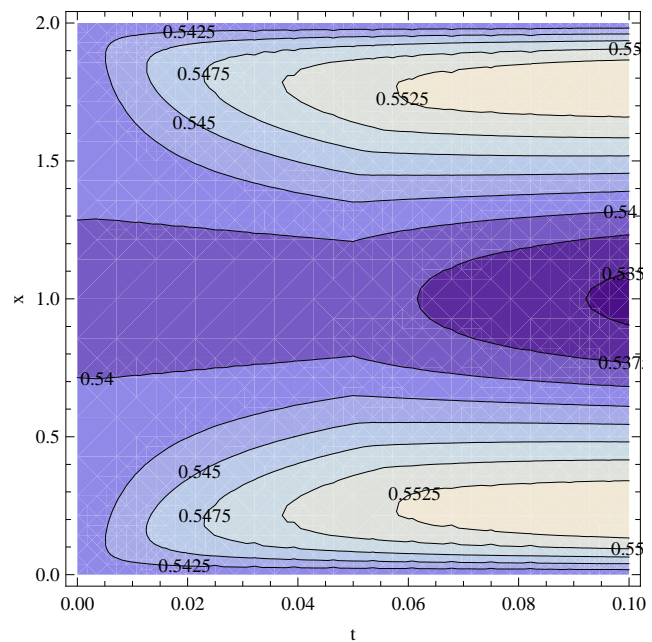

FIGURE 2.6: Case 2. $w_{k}^{*}(t, x), t \in[0, T / 10]$

The values of $J_{k}\left(v_{k}^{*}, w_{k}^{*}\right)$ for $k=0, \ldots, 8$ are : 14.8746, 14.2868, 13.7165, 13.1631, 12.6256, 12.107, $11.5939,11.2742,11.1873$.

We notice in Figures 2.3 - 2.4 and Figures 2.5 - 2.6 a better matching between the contour plots of $y_{k}^{*}(t, x)$ and $w_{k}^{*}(t, x)$, both for smaller time and for larger time. This might be explained by the initial choice $w_{0}^{*}=y_{0}^{*}$.

Acknowledgements. The author thanks the anonymous reviewers for the extremely careful lecture of this article and for the pertinent suggestions. The author acknowledges the support of the project CNCS-UEFISCDI PN-IIID-PCE-2011-3-0045 of the Romanian National Authority for Scientific Research.

\section{References}

[1] V. Arnăutu, P. Neittaanmäki. Optimal control from theory to computer programs. Kluwer Academic Publishers. Dordrecht, 2003.

[2] V. Barbu. Optimal control of variational inequalities. Pitman. Massachusets, 1984.

[3] V. Barbu. Nonlinear differential equations of monotone types in Banach spaces. Springer. New York, 2010. 
[4] C.M. Elliott, J.R. Ockendon. Weak and variational methods for moving boundary problems. Research Notes in Mathematics 59. Pitman, 1982.

[5] J.L. Lions. Quelques méthodes de resolution des problèmes aux limites non linéaires. Dunod. Paris, 1969.

[6] J.L. Lions. Contrôle des systèmes distribués singuliers. Bordas. Paris, 1983.

[7] E. Magenes. Topics in parabolic equations: Some typical free boundary problems. In: Boundary value Problems for Linear Evolution Partial Differential Equations (ed. H.G. Garnir). D. Reidel (1977), 239-312. 